years of follow-up. Pairwise post-hoc comparisons were performed by Bonferroni correction. Area Under the Receiver Operating Characteristics (AUROC) of cellular changes in FBC components to predict LOR at different time points were calculated. Latent class mixed models were used to investigate trajectories of change in cellular components of the FBC from baseline to one year. Further survival analysis to predict LOR of TNFi was done by using the latent class model and adjusted by clinical parameters, demographics and concomitant treatment. Results: 92 and 43 biologic-naïve RA patients who attained remission were included from cohorts 1 and 2 respectively. 73 (54\%) were treated with adalimumab and $62(46 \%)$ with etanercept. $87(64 \%)$ of patients lost remission over a 10 -year period with median time to LOR of 3.3 years $(95 \% \mathrm{Cl} 2.6-3.7)$.

Among the cellular components of FBC, monocyte count changes in the first year after initiation of TNFi fell significantly in those who maintained remission over the five years of follow-up, compared to those who lost remission $[p<0.001$ for all years evaluated] (Figure 1). AUROC of monocyte count change at 6 months and 12 months, to predict LOR from year 1 to year 5 (after TNFi initiation) and after 5 years, ranged between 0.70-0.90. Three latent classes were identified based on monocyte count changes over time: 1-decrease $(\mathrm{N}=41)$, 2-increase $(\mathrm{N}=7)$ and 3- no change $(\mathrm{N}=87)$. Among these latent classes, patients in class 1 , sustained remission for longer (unadjusted log-rank $p=0.001$ ) compared to the other 2 classes. Monocyte latent classes as a predictor of LOR was further tested by cox-regression, where variables (anti-citrullinated protein antibody/ACPA, concomitant methotrexate, baseline DAS28-ESR) were selected by elastic net regularisation and further adjusted by use of steroid, age and gender. Class 2 and Class 3 monocyte latent classes showed a hazard ratio (HR) of $3.15(95 \% \mathrm{Cl}$ 1.02-9.74, $p=0.046, \mathrm{n}$. of event $=6 / 7$ ) and $5.48(95 \% \mathrm{Cl} 2.6-11.57, p<0.001, \mathrm{n}$. of event $=73 / 87$ ) respectively compared to Class 1 . ACPA positivity also increased the risk of LOR with $\mathrm{HR}$ of 2.13 and $95 \% \mathrm{Cl} 1.04-4.37, p=0.04$ (n. of event $=$ $78 / 117$ ) compared to seronegative patients ( $n$. of event $=9 / 18$ ).

Conclusion: In seropositive RA patients treated with $\mathrm{TNFi}$, a reduction of total monocyte count in the first year was associated with maintenance of remission in subsequent years. Further studies are required to determine whether these effects are specific to TNFi or reflect sustained remission in RA irrespective of therapy.

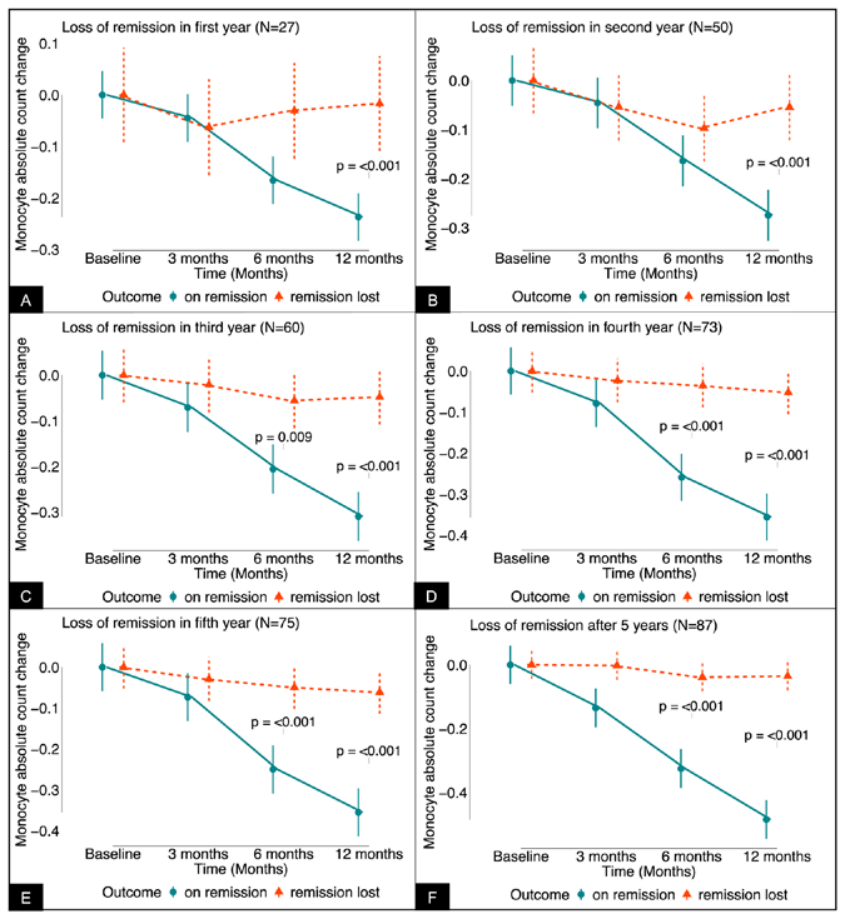

Figure 1 (A-F). Mean change (with 95\% confidence interval) in absolute count of monocyte after initiation of tumour necrosis factor inhibitor grouped by years of follow-up and stratified by loss of remission. $\mathrm{N}=$ number indicate cumulative number of patients who lost remission by the end of each year.

Acknowledgements: Muhammad Shipa is funded by Versus Arthritis. Su-Ann Yeoh is funded by the Royal College of Physicians, Rosetrees Trust, NIHR University College London Hospitals Biomedical Research Centre, UCLH Charities, and Versus Arthritis. Michael Ehrenstein is supported (in part) by the University College London Hospital Biomedical Research Centre. None of the funding bodies have been involved in preparation of this manuscript.

Disclosure of Interests: None declared

DOI: 10.1136/annrheumdis-2021-eular.312

\section{POS0441 DEVELOPMENT OF RHEUMATOID ARTHRITIS AMONG ANTI-CITRULLINATED PROTEIN ANTIBODIES POSITIVE ASYMPTOMATIC INDIVIDUALS: A PROSPECTIVE OBSERVATIONAL STUDY}

S. Mizuki ${ }^{1}$, K. Horie ${ }^{1}$, K. Imabayashi ${ }^{1}$, K. Mishima ${ }^{2}$, K. Oryoji ${ }^{1}{ }^{1}$ Matsuyama Red Cross Hospital, The Center for Rheumatic Diseases, Matsuyama, Japan; ${ }^{1}$ Matsuyama Red Cross Hospital, The Center for Rheumatic Diseases, Matsuyama, Japan

Background: In the idividuals with genetic and enviromental risk factors, immune events at mucosal surfaces occur and may precede systemic autoimmunity. Anti-citrullinated protein antibodies (ACPA) are present in the serum for an average of 3-5 years prior to the onset of rheumatoid arthritis (RA) during an asymptomatic period. In ACPA-positivite individuals, the additional presence of RA-related risk factors appears to add significant power for the development of RA. To date, there have been few reports in which clinical courses of ACPA-positive asymptomatic individuals were investigated prospectively.

Objectives: To observe the clinical time course of ACPA-positive healthy population for the development of RA.

Methods: Healthy volunteers without joint pain or stiffness, who attended the comprehensive health screening of our hospital, were enrolled in this prospective observational study. The serum ACPA levels were quantified by Ig-G anti-cyclic citrullinated peptide enzyme-linked immunosorbent assay with levels $>4.4 \mathrm{U} / \mathrm{mL}$ considered positive. ACPA-positive subjects were followed by rheumatologists of our department clinically or a questionnaire sent by mail for screening to detect arthritis.

Results: 5,971 healthy individuals without joint symptons were included. Ninty-two (1.5\%) were positive for ACPA. Of these, 19 (20.7\%) developed RA and two were suspected as RA by mail questionnaire. Their average age were 58 -years, and women were $68 \%$. The average duration between the date of serum sampling and diagnosis was 10.7 months. ACPA-positive individuals who developed to RA had higher serum ACPA and Ig-M rheumatoid factor levels than ACPA-positive individuals who did not ( $P$ value by Mann-Whitney $U$ test: 0.002 , 0.005 , respectively).

Conclusion: Among ACPA-positive asymptomatic individuals, 20\% developed $\mathrm{RA}$. The higher titer of ACPA and Ig-M rheumatoid factor levels are risk factors for devoloping RA.

Disclosure of Interests: None declared

DOI: 10.1136/annrheumdis-2021-eular.344

\begin{tabular}{|l|l|}
\hline POS0442 & RELATIONSHIP BETWEEN PARAOXONASE-1 \\
GENOTYPE, ACTIVITY AND MAJOR ADVERSE \\
CARDIOVASCULAR EVENTS IN PATIENTS WITH \\
RHEUMATOID ARTHRITIS RECEIVING TOFACITINIB
\end{tabular}

C. Charles-Schoeman ${ }^{1}$, C. Hyde ${ }^{2}$, S. Guan ${ }^{3}$, N. Parikh ${ }^{1}$, J. Wang ${ }^{1}$

A. Shahbazian ${ }^{1}$, L. Stockert ${ }^{4}$, J. Andrews ${ }^{4} .{ }^{1}$ University of California, Department of Medicine, Los Angeles, CA, United States of America; ${ }^{2}$ Pfizer Inc, Inflammation and Immunology, Groton, CT, United States of America; ${ }^{3}$ Pfizer Inc, Inflammation and Immunology, Cambridge, MA, United States of America ${ }^{4}$ Pfizer Inc, Inflammation and Immunology, Collegeville, PA, United States of America

Background: Paraoxonase-1 (PON1) is a high-density lipoprotein (HDL)-associated enzyme with paraoxonase, lactonase and arylesterase activities. ${ }^{1}$ PON1 contributes to the antioxidant properties of $\mathrm{HDL}$, and is being investigated for its atheroprotective properties. ${ }^{1}$ Patients (pts) with rheumatoid arthritis (RA) who are homozygous for the RR genotype of the Q192R gene polymorphism on PON1 (rs662) have increased paraoxonase activity, and lower risk of carotid plaques, vs those with $Q Q$ or $Q R$ genotypes. ${ }^{2}$ Tofacitinib is an oral Janus kinase inhibitor for the treatment of RA.

Objectives: To investigate the relationship between PON1 genotype/activity and risk of major adverse cardiovascular events (MACE) in the tofacitinib RA clinical programme.

Methods: In this post hoc analysis, data were pooled from pts enrolled in nine Phase 2/3 studies of tofacitinib in RA. Enzyme activities in pt plasma samples were measured at individual study baseline $(B L)$ and at follow-up visits using three substrates: paraoxon (paraoxonase activity), dihydrocoumarin (lactonase activity) and phenylacetate (arylesterase activity). The effect of the PON1 Q192R genotype ( $\mathrm{QQ}, \mathrm{QR}$ or $\mathrm{RR}$ ) on $\mathrm{BL}$ paraoxonase/lactonase/arylesterase activity was assessed using linear regression for each study, with age and sex as covariates, and then fixed-effect meta-analysis assessed effects across studies. The risk of MACE by enzyme activity was determined using Cox proportional hazards regression stratified by clinical studies. Univariate regression against $B L$ enzyme activity and other risk factors, as well as both minimally and fully adjusted multivariable regressions against time-varying enzyme activity, are presented.

Results: The analysis included 1969 pts with RA who received $\geq 1$ dose of tofacitinib and had PON1 activity measures available at BL; 39 pts had $\geq 1$ 